\title{
Foveal vision power errors induced by spectacle lenses designed to correct peripheral refractive errors
}

Sergio Barbero', Miguel Faria-Ribeiro²

1. Instituto de Optica, Consejo Superior de Investigaciones Cientificas (IO, CSIC), Madrid, Spain

2. Clinical \& Experimental Optometry Research Lab (CEORLab), Center of Physics, University of Minho, Braga, Portugal

Published as final edit form as: Barbero $S$ \& Faria-Ribeiro M. Foveal vision power errors induced by spectacle lenses designed to correct peripheral refractive errors. Ophthalmic Physiol Opt 2018. https://doi.org//10.1111/opo.12442

Keywords: Radial Refractive Gradient (RRG), spectacles, spectacles lens design, spectacles power errors

Correspondence: Sergio Barbero

E-mail address: sergio.barbero@csic.es

Received: 3 October 2017;

Accepted: 28 December 2017

\section{Abstract}

Purpose: The so-called Radial Refractive Gradient (RRG) spectacles are lenses specifically designed to minimize peripheral hyperopic defocus typically found in conventional spectacles. Our goals are: 1) to demonstrate a method to design such lenses; and 2) to quantify the exact foveal vision power errors induced by them.

Methods: The design procedure is based on a point-by-point sequential surface construction algorithm that designs a front aspheric surface (back surface is spherical) to achieve a given overall tangential focal length of the lens. A peripheral refraction model was built based on average peripheral refractive errors from a set of eyes. We designed four negative lenses with optical 
powers: - 2.5 D, $-5 \mathrm{D},-7.5 \mathrm{D}$ and $-10 \mathrm{D}$, so that the tangential focal length of the lens matches the retinal conjugate surface.

Results: The lenses induce very small sagittal power errors in a wide range of off-axis field angles $\left(30^{\circ}\right)$, solving the problem of peripheral hyperopic defocus. However, such designs introduce non-negligible mean power errors (above $0.25 \mathrm{D}$ from $7^{\circ}, 6.8^{\circ}, 7.1^{\circ}$ and $7.8^{\circ}$ for the $-2.5 \mathrm{D},-5 \mathrm{D},-7.5 \mathrm{D}$ and $-10 \mathrm{D}$ lenses, respectively) for foveal vision in a rotating eye.

Conclusion: Our results show the unavoidable errors introduced by RRG spectacles when used for dynamic foveal vision. The described method offers valuable information towards determining the best trade-off between controlling power errors for peripheral and foveal vision.

\section{Introduction}

With the prevalence of myopia rising dramatically, a global myopia epidemic is imminent. As less myopic generations are replaced by younger and more myopic generations, the population prevalence of myopia in adults will reach this global scenario in no more than two to three decades. ${ }^{1}$ High myopia is associated with permanent visual impairment that increases in a non-linear way with the increase in eye elongation, ${ }^{2}$ making it, along with its associated agerelated blinding pathologic lesions, a threat for a significant part of the future generations. ${ }^{3}$ This takes even more worrying proportions when considering that the early onset of myopia, seen in the younger generations, leaves more time for progression at higher rates.

Although the mechanism behind myopia progression is still open to debate, evidence based from animal studies suggests that peripheral hyperopic defocus exacerbates myopia progression, while inducing myopic peripheral defocus can slow myopia progression. ${ }^{4,5}$ Controversially, other studies suggest that hyperopic defocus is more a consequence than a cause of myopia. ${ }^{6}$ Based on the former premise, several methods have been proposed to avoid peripheral hyperopic defocus. Some of them rely on the use of orthokeratology, ${ }^{7,8}$ rigid $^{9}$ or 
soft bifocal contact lenses, ${ }^{10}$ and others in specially designed spectacle lenses that have been named Radial Refractive Gradient (RRG) spectacles. ${ }^{11}$

Conventionally, single-vision spectacles aim at correcting the refractive error at foveal vision with a rotating eye. Thus, the challenge of the optical designer is to select the lens surface profiles that reduce the oblique power errors (mean power error and astigmatism) for different gaze directions. Following such design as target implies that peripheral vision is not controlled, so it is not surprising that they introduce some amount of relative peripheral hyperopia. ${ }^{11,12}$ This is the reason why the first RRG spectacles designs were proposed, although previous attempts to minimize peripheral power errors in spectacles design were focused more on the role of peripheral optics in visual detection, rather than on controlling myopia progression. ${ }^{13}$

\section{State of the art in RRG designs}

As for current RRG existing designs, most of available information is found in patent literature. One of the earliest proposals was that of Smith et al. ${ }^{14}$ They prioritized mean power error correction over oblique astigmatism, particularly by locating both the tangential and the sagittal focal surfaces in front of the retina or setting the sagittal focal surface at the retina.

Unavoidably RRG designs may significantly deteriorate the optical quality for foveal vision at gaze directions different from the primary line of sight. To avoid this problem, a trade-off design philosophy, aim at partially controlling power errors for peripheral and foveal vision simultaneously, has been adopted. The design idea is to divide the spectacles lens area in two different parts, one with a central area around the primary position where the eye is assumed to be confined in its rotatory movements, and a peripheral area used by the eye to perceive extra-foveal images. The designs following this approach minimize refractive power errors for different gaze directions in the central area, and peripheral refractive errors, for the eye located at primary position of gaze, in the outer area. Following this design idea, Ho et al. ${ }^{15}$ proposed a variation over their previous, already discussed, patent. ${ }^{14}$ Varnas et al. patents, ${ }^{16-18}$ which are the basis of the so-called MyoLens ${ }^{\circledR}$ by Zeiss, also use this philosophy. Yet 
another proposal, following this idea, was presented by Tabernero et al., ${ }^{11}$ where a central area (6 $\mathrm{mm}$ diameter) was designed with constant power, and a peripheral area with a varying refractive profile around one dioptre for every 10 degrees.

Goals

Beyond the debate of whether peripheral hyperopia is a cause or a consequence of myopia, a serious evaluation of RRG lenses requires a precise quantitative understanding of the foveal vision power errors induced by RRG spectacles. Unfortunately, not much work has been published on this issue. At this respect, a crucial work was that of Atchison, ${ }^{19}$ who using third-order aberration theory found that $-4 D$ and $-6 D$ spherical spectacles can induce up to $0.65 \mathrm{D}$ and $1.01 \mathrm{D}$ of tangential error, respectively for 35 degrees of ocular rotation. Atchison ${ }^{19}$ also extended his analysis using ray tracing (with commercial software Zemax) over third-order analytical solutions, particularly comprising conic surfaces. This is an important point because, as it is well known by optical designers, exact generalized ray tracing computations show results considerable different to those predicted by third order aberration theory. ${ }^{20}$

In this work, we continue with Atchison's attempt introducing an important novelty. We first show how to design RRG lenses without restriction to thirdorder theory and second, through computer simulations, quantify the exact foveal vision power errors induced by such lenses.

\section{Methods}

\section{RRG spectacle design method}

In the same way that is done in conventional spectacle lens design, the eye is reduced to a reference point, ${ }^{21}$ which means that the image forming elements of the eye (cornea and crystalline lens) are not considered. However, whereas in foveal vision spectacles design (rotating eye) this reference point is the centre of rotation of the eye, in peripheral vison (stationary eye) it is the centre 
of the entrance pupil of the eye ${ }^{13,19}$ or alternatively the nodal point of the eye, ${ }^{22}$ which has the advantage of avoiding distortion effects. We chose the entrance pupil as the more natural selection in off-axis optical systems analysis.

Figure 1 represents the geometrical elements involved in spectacle lens design for a stationary eye. $V$ denotes the back-vertex point, i.e. the intersection of the optical axis with the posterior lens surface. $E$ is the centre of the entrance pupil. The sphere whose rotation centre is located at $E$ and whose radius of curvature is given by the segment VE is called vertex sphere. For the distance from spectacles vertex to the centre of the entrance pupil, we used the value 15 $\mathrm{mm} .{ }^{19}$ The back vertex focal distance of the lens is denoted by $f$. The far point sphere (vertex point F) is defined as concentric with the vertex sphere and having a radius given by the sum of VE and $\boldsymbol{f}$. The far point sphere is denoted by FPS (black curve) in Fig. 1.

For each off-axis beam of light (incident angle $\alpha$ ) there is a point at the vertex sphere (A at Fig. 1). At each of these points it is possible to compute a localized quadratic wavefront ${ }^{20}$ or local dioptric matrix ${ }^{23}$ characterizing the mean power and cylinder of the off-axis beam. The art of designing spectacle lenses consists of finding lens surfaces that ensure a specific target of these quantities. Particularly, if the target is a uniform optical power then each local beam of light must perfectly focalize at the corresponding point at far point sphere (B). 


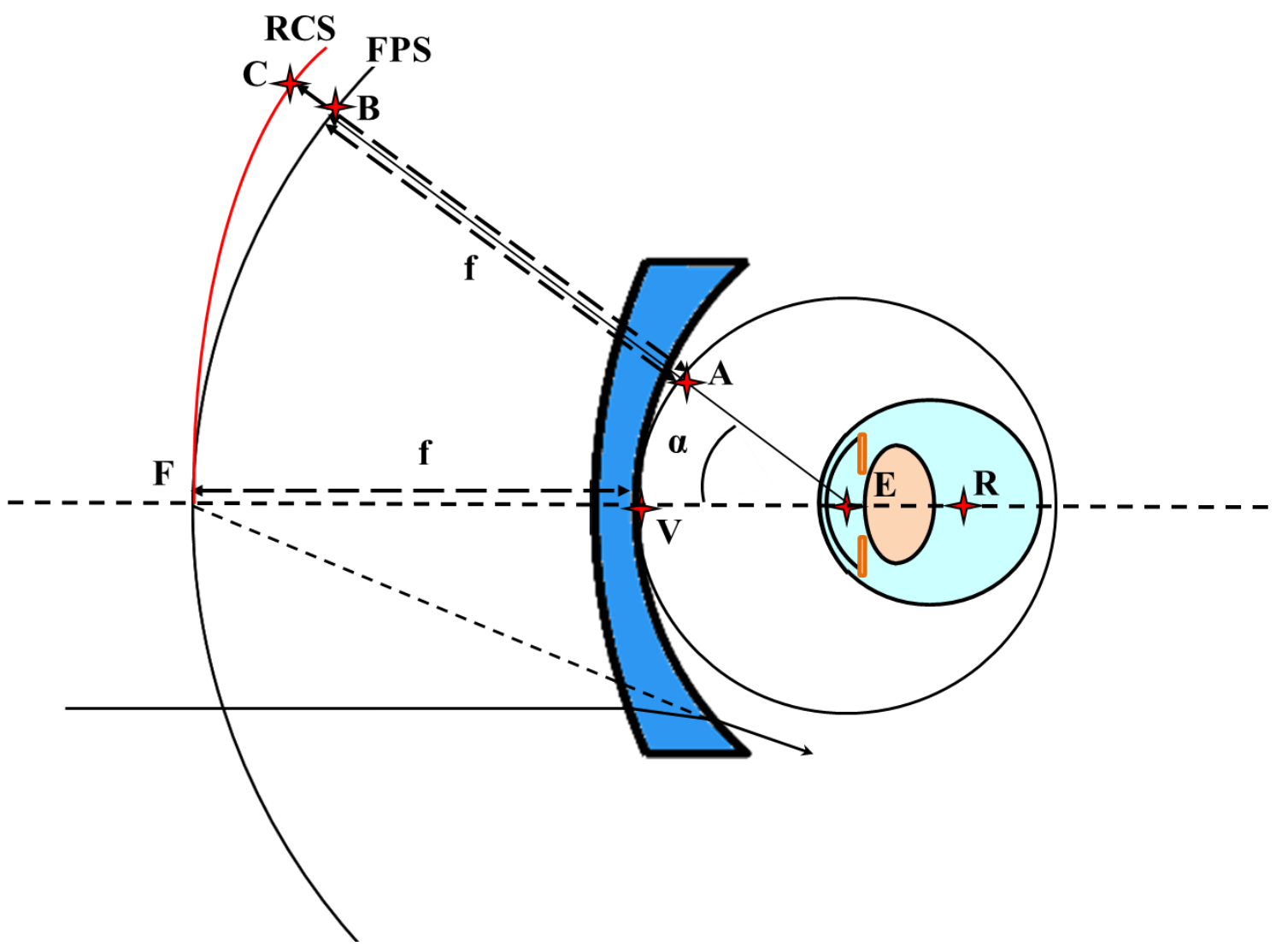

Fig. 1. Scheme showing the geometrical parameters used in spectacle lens design for a stationary eye. Far point sphere (FPS) and retinal conjugate surface (RCS) are depicted with black and red solid lines, respectively.

To take into account peripheral refraction changes means that the focalization target is no longer the far point sphere. Thus, we have to define a new surface (it has been coined the retinal conjugate surface ${ }^{22}$ ) which considers the power error of the myopic eye at different eccentricities. The relative peripheral hyperopic error of the eye implies the need for longer (in absolute terms) focal lengths; thus, the retinal conjugate surface (RCS) is located farther from the spectacles than the far point sphere, as shown in Fig. 1 (red solid line and point $\mathrm{C})$.

\section{Retinal conjugate surface}

To characterize the retinal conjugate surface, for our design problem, the peripheral refraction change with incident angle $\alpha$ is required. This information 
may be obtained either from an eye model or from empirical data extracted from a set of subjects. In this work, we opted for the latter approach.

Peripheral refraction can be obtained using an open field autorefractor, like the Grand Seiko (Grand Seiko Co., Ltd., Hiroshima, Japan). This instrument operates on the image size principle in which refraction is linearly related to the angular size of the image reflected by the retina. Osuagwu ${ }^{24}$ studied the validity of this approach and concluded that the autorefractor's peripheral refraction measurements are valid for horizontal and vertical field meridians, but not for oblique field meridians.

The clinical data used in this study were obtained from a previous work, ${ }^{25}$ where the peripheral refraction data from 55 myopic patients was measured along the horizontal field using the Grand Seiko WAM-5500 open field autorefractor, but for this work only the mean relative data from the most hyperopic horizontal semi-meridian (temporal retina hemifield) were used. The relative peripheral refraction, which can be interpreted as the patient's off-axis refraction when compensating for his central refractive error, was obtained by subtracting the central refraction from the peripheral refraction, using vector form notation $\left(\mathrm{M}, \mathrm{J}_{0}\right.$ and $\left.\mathrm{J}_{45}\right){ }^{26}$ After subtracting central refractive error from peripheral refraction, $\mathrm{J}_{45}$ vector component becomes of negligible value (along the horizontal visual field). These small values arise mainly due to alignment problems from the autorefractor, although it can also arise from irregularities in the optical components (cornea mainly). Even so, for simplicity we assumed that $J_{0} \approx \sqrt{J_{0} \times J_{0}+J_{45} \times J_{45}}$, so that the tangential and sagittal power errors along the horizontal visual field can be defined as $M+J_{0}$ and $M-J_{0}$, respectively.

By applying least squares fitting of overall data with $2^{\text {th }}$ degree polynomials, the following equations were obtained and used to describe the difference in tangential $\left(\mathrm{E}_{\mathrm{T}}(\alpha)\right)$ and sagittal $\left(\mathrm{E}_{\mathrm{S}}(\alpha)\right)$ power with respect to that at the primary line of sight as function of field angle $\alpha$ :

$$
\begin{aligned}
& E_{T}(\alpha)=-0.00031 \alpha^{2}-0.028 \alpha \quad\left(\mathrm{R}^{2}=0.90\right) \\
& E_{S}(\alpha)=0.00081 \alpha^{2}-0.00024 \alpha \quad\left(\mathrm{R}^{2}=0.99\right)
\end{aligned}
$$


Figure 2 shows $\mathrm{ET}_{\mathrm{T}}(\alpha)$ and $\mathrm{Es}_{\mathrm{s}}(\alpha)$ as function of peripheral field angle.

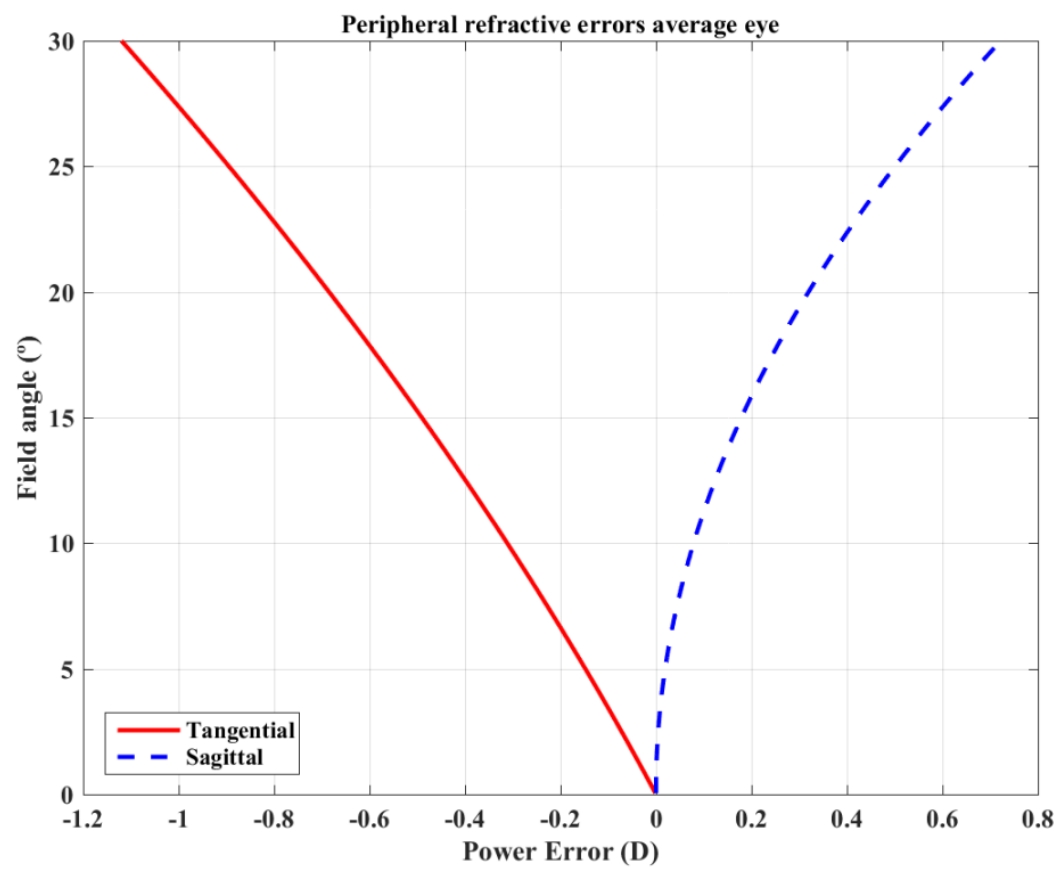

Fig. 2. Tangential $\left(E_{T}(\alpha)\right)$ and sagittal $(E s(\alpha))$ relative peripheral power errors (from 55 myopic patients) as function of field angle.

Tangential and sagittal spectacles powers as function of angle were denoted by $\operatorname{PT}(\alpha)$ and $P_{S}(\alpha)$, respectively. The power differences with respect to primary line of sight are $\Delta P_{T}(\alpha)=P_{T}(\alpha)-P(\alpha=0)$ and $\Delta P_{S}(\alpha)=P_{S}(\alpha)-P(\alpha=0)$. Now, we assume that eye peripheral power error correction is achieved by direct addition (or subtraction) of peripheral power changes induced by spectacles. ${ }^{13}$ Thus,

$$
R_{T}(\alpha)=E_{T}(\alpha)+\Delta P_{T}(\alpha)
$$

and

$$
R_{S}(\alpha)=E_{S}(\alpha)+\Delta P_{S}(\alpha)
$$

where $R_{T}(\alpha)$ and $R_{S}(\alpha)$ are the tangential and sagittal errors of the eye, respectively, when spectacles for peripheral correction are used.

To completely correct the peripheral errors of the eye, it would be necessary to cancel both $R_{T}(\alpha)$ and $R_{S}(\alpha)$ in Eq. (3) and (4), a goal which, in principle, 
requires two aspheric surfaces. ${ }^{27}$ Restricted to designs involving a single aspherical surface, either the tangential or the sagittal focus could be corrected. ${ }^{28}$ Nevertheless, to compensate any of the eye's relative peripheral hyperopia only the sagittal relative error needs be cancelled (see figure 2). This might be achieved by designing a lens where:

$$
P_{S}(\alpha)=\mathrm{P}(\alpha=0)-E_{S}(\alpha)
$$

However, our design procedure sets the lens surface in order to get a specific $\mathrm{P}_{T}(\alpha){ }^{29}$ Thus, the remaining problem is how to determine $\mathrm{P}_{T}(\alpha)$ values that satisfy Eq. (5). This can be done if somehow, we relate $\operatorname{PT}_{T}(\alpha)$ to $\operatorname{Ps}_{S}(\alpha)$.

To obtain an analytical expression for this relation is probably unattainable using a thick aspheric lens. However, it is known that for an ellipsoid of revolution, tangential and sagittal powers are related through ${ }^{30}: \mathrm{PT}_{\mathrm{T}}=\mathrm{Ps}^{3} / \mathrm{P}^{2}$, being $P_{0}$ the on-axis power at the vertex. Assuming that the power changes of the lenses being designed follow approximately the same rule, Eq. (5) can be solved for $\mathrm{PT}_{\mathrm{T}}(\alpha)$ :

$$
P_{T}(\alpha)=\mathrm{P}(\alpha=0)-\frac{E_{S}(\alpha)^{3}}{P(\alpha=0)^{2}}
$$

Finally, the retinal conjugate surface needed for the design is simply the inverse of $\mathrm{P}_{\mathrm{T}}(\alpha)$, as given by Eq. (7).

\section{Optical design procedure}

Conventional ophthalmic lens design is based on finding the optimal parameters of a lens surface descriptor through an optimization algorithm that minimizes a given merit function. Here, instead, we used a point-by-point sequential surface construction algorithm published elsewhere. ${ }^{29}$ Specifically, the algorithm designs a front aspheric surface (back surface is spherical) to achieve a given overall tangential focal length of the lens.

For completeness, we briefly review the major steps of the design procedure:

1. A paraxial pre-design is selected (lens material, central thickness, and apical curvatures of anterior and posterior lens surfaces). 
2. A recurrence algorithm constructs point-by-point the lens front surface using quadratic patches as local descriptors.

3. The front surface constructed as a set of points is fitted (least squares solution) to an aspheric surface described by:

$$
z(\rho)=\frac{c \rho^{2}}{1+\sqrt{1-(Q+1) c^{2} \rho^{2}}}+\sum_{i=1}^{6} K_{i} \rho^{2 i+2}
$$

where $\rho$ is the radial coordinate, $c$ is the vertex curvature: $c=1 / R$, being $R$ the vertex radius of curvature, $Q$ is the conic parameter $\left(Q=-e^{2}\right)$, and $K_{i}$ are the higher order coefficients of the aspheric surface.

\section{Results}

\section{Design examples}

The design analyses were done for a set of four negative lenses with optical powers of $-2.5 D,-5 D,-7.5 \mathrm{D}$ and $-10 \mathrm{D}$. CR-39 polymer ( $n=1.498)$ was chosen as the lens material and the central thickness was fixed to $1.6 \mathrm{~mm}$. Base curves (instead of simply using the third-order solution) were optimized to obtain best values for each power. The designs analysis was done with a maximum of $30^{\circ}$ of angle of incidence. Although eye rotations can be up to $45^{\circ}$, eye-head coordination, in natural conditions, makes eye turn (at final gaze positions) larger than $20^{\circ}$ very rare. ${ }^{31}$

The final geometrical parameters of the lenses are shown in Table 1. Additionally, the surface profiles are depicted in Figure 3. $R_{f}$ and $R_{b}$ are the front and back radii of curvature, respectively. $Q_{f}$ and $K_{i}$ are the conic parameter and the higher order coefficients of the front surface, respectively.

\begin{tabular}{|l|lllllllll|}
\cline { 2 - 9 } \multicolumn{1}{c|}{} & $\mathrm{R}_{\mathrm{f}}$ & $\mathrm{R}_{\mathrm{b}}$ & $\mathrm{Q}_{\mathrm{f}}$ & $\mathrm{K}_{1}$ & $\mathrm{~K}_{2}$ & $\mathrm{~K}_{3}$ & $\mathrm{~K}_{4}$ & $\mathrm{~K}_{5}$ & $\mathrm{~K}_{6}$ \\
\hline$-2.5 \mathrm{D}$ & 83.00 & 58.32 & $1.03 \mathrm{E}-3$ & $5.57 \mathrm{E}-6$ & $-1.60 \mathrm{E}-8$ & $4.18 \mathrm{E}-11$ & $-0.84 \mathrm{E}-13$ & $1.09 \mathrm{E}-16$ & $-0.66 \mathrm{E}-19$ \\
& & & & & & & & & \\
\hline$-5 \mathrm{D}$ & 62.25 & 38.11 & $1.19 \mathrm{E}-4$ & $5.15 \mathrm{E}-6$ & $-0.98 \mathrm{E}-8$ & $2.02 \mathrm{E}-11$ & $-0.31 \mathrm{E}-13$ & $0.30 \mathrm{E}-16$ & $-0.12 \mathrm{E}-19$ \\
\hline
\end{tabular}




\begin{tabular}{|l|lllllllll|}
\hline$-7.5 \mathrm{D}$ & 55.33 & 30.02 & $0.93 \mathrm{E}-4$ & $4.35 \mathrm{E}-6$ & $-0.66 \mathrm{E}-8$ & $0.75 \mathrm{E}-11$ & $0.10 \mathrm{E}-13$ & $-0.57 \mathrm{E}-16$ & $0.66 \mathrm{E}-19$ \\
\hline$-10 \mathrm{D}$ & 49.80 & 24.77 & $1.73 \mathrm{E}-4$ & $3.17 \mathrm{E}-6$ & $-0.24 \mathrm{E}-8$ & $-1.73 \mathrm{E}-11$ & $1.15 \mathrm{E}-13$ & $-2.30 \mathrm{E}-16$ & $2.30 \mathrm{E}-19$ \\
& & & & & & & & & \\
\hline
\end{tabular}

Table 1. Design parameters of $-2.5 \mathrm{D},-5 \mathrm{D},-7.5 \mathrm{D}$ and $-10 \mathrm{D}$ lenses. $R_{f}$ and $R_{b}$ units are $\mathrm{mm} . \mathrm{K}_{\mathrm{i}}$ units are $m m^{-(2 i+1)} Q_{\mathrm{f}}$ is unitless.

It should be noticed that the lens anterior surface, computed by the point-bypoint recurrence algorithm, was only calculated for a $30^{\circ}$ maximum field (lens diameter of about 30 millimetres) and without imposing constrains on the edge thicknesses and curvatures. Additionally, we point out that the radii of curvature slightly differ from the required values to match the nominal paraxial back vertex power. This slight discrepancy is a collateral effect of the design procedure and its posterior data point fitting.

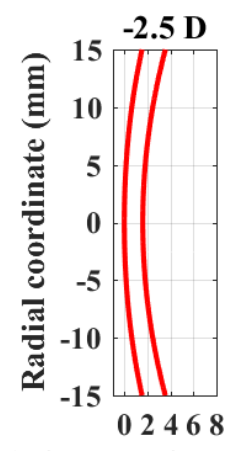

Axial coordinate (mm)

(a)

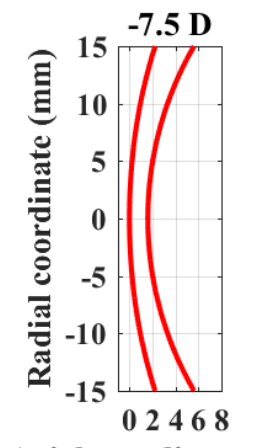

Axial coordinate (mm)

(c)

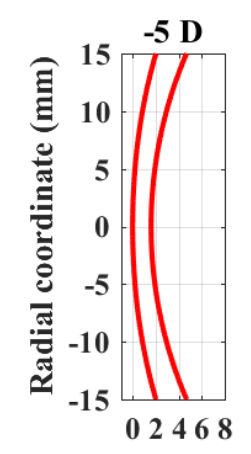

Axial coordinate (mm)

(b)

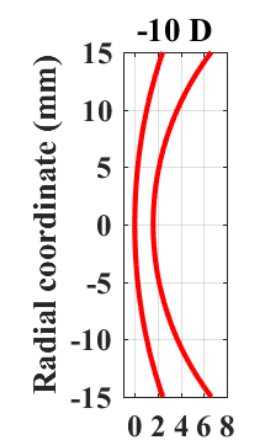

Axial coordinate ( $\mathrm{mm})$

(d)

Fig. 3. Design profiles of lenses given in Table 1. 
$\operatorname{PT}_{T}(\alpha)$ and $\operatorname{Ps}(\alpha)$ of designed lenses were computed following a procedure described in a previous paper. ${ }^{29}$ The tangential and sagittal power errors were then computed using Eqs. (3) \& (4). Figure 4 shows the resulting $\mathrm{R}_{\mathrm{T}}(\alpha)$ and $R_{s}(\alpha)$ as function of field angle. For all designs and evaluated angles (up to $\left.30^{\circ}\right)$, the eye's sagittal power errors were strongly compensated, which resulted in an $R_{s}(\alpha)$ value contained between $-0.187 \mathrm{D}$ and $+0.075 \mathrm{D}$. As expected from the relationship between $\mathrm{Ps}$ and $\mathrm{PT}$ described in Eq. (7), tangential power errors increase much more rapidly as a function of field angle.

Peripheral analysis (Static design)
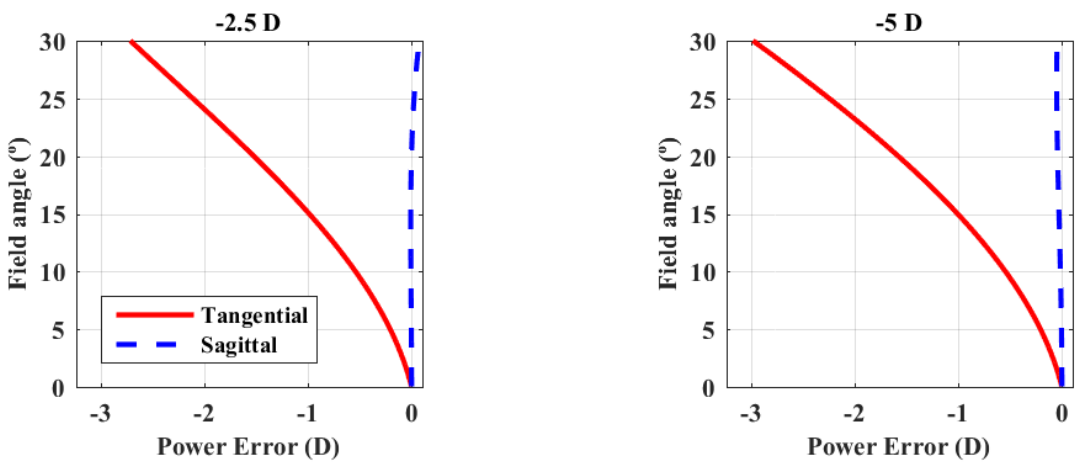

(a)

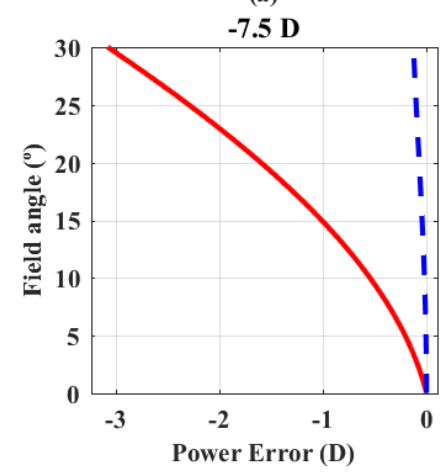

(c)

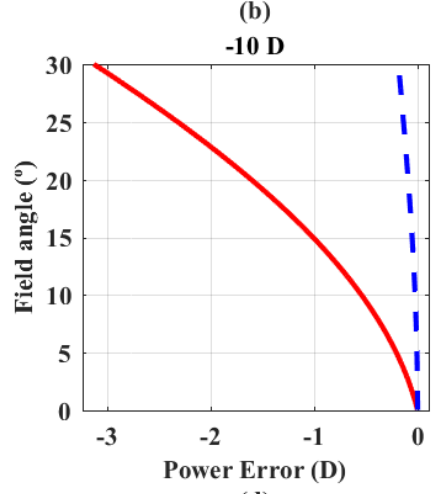

(d)

Fig. 4. Tangential (red solid line) and sagittal (blue dashed line) power errors (D) as function of field angle (degrees) for designs (a) $-2.5 \mathrm{D}$, (b) $-5 \mathrm{D}$, (c) $-7.5 \mathrm{D}$ and (d) -10 D. Errors are computed for extra-foveal vision in a static eye.

The same problem applies when the eye rotates behind the lens, with tangential power errors increasing more rapidly as function of gaze direction, as shown in Figure 5. For this case, instead of the centre of the pupil, the centre of rotation of the eye, denoted by $\mathrm{R}$ in Figure 1 and located $27 \mathrm{~mm}$ behind the 
vertex sphere, was used. Mean errors are below $0.25 \mathrm{D}$ only up to $7^{\circ}, 6.8^{\circ}, 7^{\circ} 1^{\circ}$ and $7.8^{\circ}$ for the $-2.5 \mathrm{D},-5 \mathrm{D},-7.5 \mathrm{D}$ and $-10 \mathrm{D}$ lenses, respectively.

The change in power as function of angle is non-linear for both evaluations (static and dynamic eye). This phenomenon, typically found in ophthalmic lens design $^{32}$ is caused by the non-linear terms of the generalized Coddington equations and the thick nature of real lenses.

\section{Eye rotation}
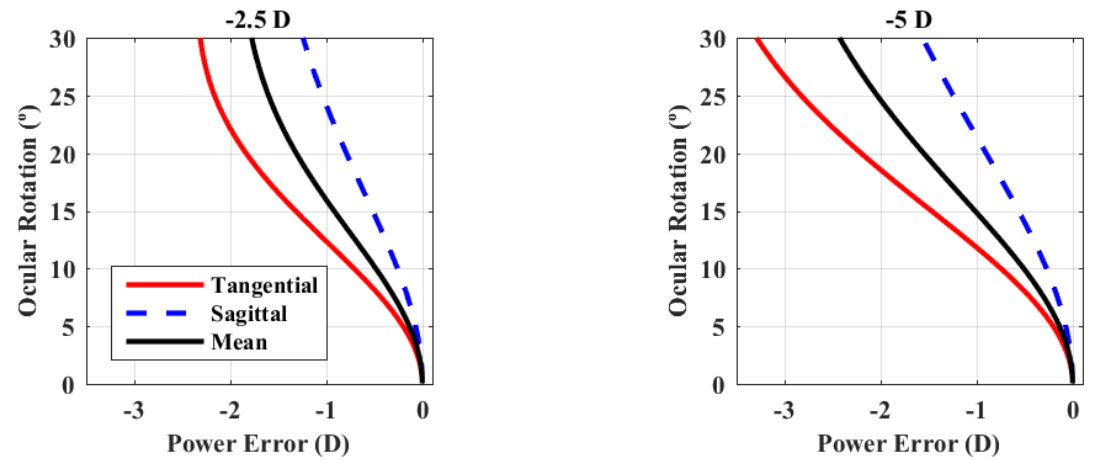

(a)

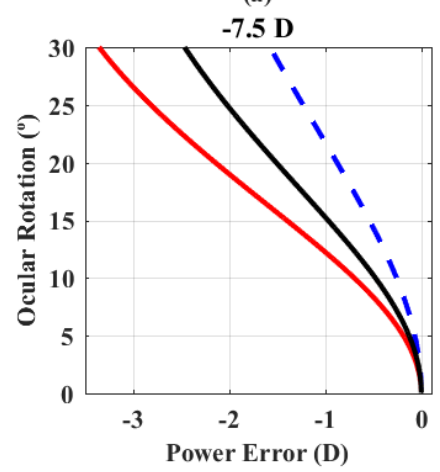

(c)

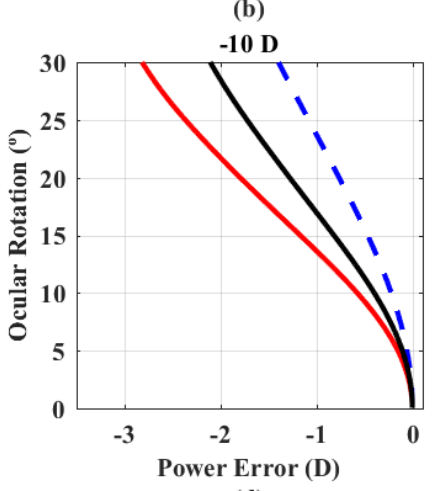

(d)

Fig. 5. Tangential (red solid line), sagittal (blue dashed line) and mean (black solid line) power errors (D) as function of ocular rotation (degrees) for designs (a) -2.5 D, (b) -5 D, (c) -7.5 D and (d) -10 D. Errors are computed for foveal vision in a rotating eye.

\section{Discussion}

Our design method is capable of generating lenses that approximately compensate the sagittal power errors of the eye in a wide range of off-axis field angles $\left(30^{\circ}\right)$, solving the problem of relative peripheral hyperopic defocus of the 
sagittal foci. We note that by heuristically fine tuning of Eq. (7) we could even force more negative sagittal power errors. However, due to the relationship between sagittal and tangential spectacles powers, approximated in this work by Eq. (7), the off-axis astigmatism of the eye increases from about $1.85 \mathrm{D}$ in the naked eye up to about $3.0 \mathrm{D}$ at $30^{\circ}$ field angle while wearing the lenses. Although peripheral resolution acuity perceived by the visual system is limited by the exponential decrease in the sampling of cones and increased receptive fields size with eccentricity, ${ }^{35}$ grating detection acuity depends strongly on optical blur in the periphery. ${ }^{36}$ Thus, at peripheral retinal locations, contrast sensitivity for detection tasks while wearing the peripheral lenses is expected to be better for radially orientated, compared to non-radially orientated, spatial frequencies, due to the eye's uncompensated off-axis tangential error.

Unavoidably such designs also introduce non-negligible power errors for foveal vision when the eye rotates, which reduce visual acuity especially during distance viewing and may affect natural eye movement patterns, by diminishing the amount of eye rotations and making them more irregular, ${ }^{33}$ an effect that has already been reported in progressive addition lenses (PALs). ${ }^{34}$

Finally, we note that the great variability on the peripheral refractive profiles between subjects ${ }^{37}$ could seriously limit the feasibility of RRG spectacles. As already warned by Smith et al., ${ }^{13}$ designing spectacles for peripheral vision is more challenging than for foveal vision, because peripheral vision is more sensitive to individual differences between eyes, mainly because of the changes in the retinal surface shape. In this work, we designed four negative lenses with different back vertex powers that were optimized for retinal conjugate surfaces that departed from the same relative peripheral errors, obtained from the average sagittal power errors of 55 low and medium myopes (from -0.60 to $5.80 \mathrm{D}$; median = -2.27 D). Although high myopes may exhibit higher values of relative peripheral hyperopia, due to steeper retinal surface shapes, ${ }^{25}$ the degree of the hypermetropic shift along the horizontal visual field does not appear to be much influenced by the amount of foveal myopia beyond about 4 $D,{ }^{38}$ which makes our approach reasonable. It should also be noted that peripheral refractive errors vary with meridian and the results on which the 
designs were based were for the horizontal meridian. ${ }^{38}$ One possible strategy to guarantee that all meridians are compensated for relative peripheral hyperopia would be to define the retinal conjugate surface based on the data of the most relative hyperopic meridian. Some authors have also proposed to take into account this effect by modifying the equations that estimate the peripheral power errors, adding the contributions of the retinal shape through some specific models. ${ }^{19}$ This information could lead to customized design spectacles. However, even in this best-scenario, our results show the unavoidable errors introduced by RRG designs when used for dynamic foveal vision.

In summary, our results support the need for a trade-off between controlling power errors for peripheral and foveal vision. One solution (already proposed, as mentioned in the introduction) is to divide the design in two areas: 1) a central area where the design is done using a rotating eye; 2) an outer area where the surface designs are the ones obtained by our procedure. The angle of incidence that marks the separation between both areas could be determined by a threshold value in the tolerable foveal errors; say, for instance, those areas below $0.25 \mathrm{D}$ in Fig. 5. Another possibility, which is worth exploring, would be to modify our design procedure to include a hybrid metric that simultaneously considers both power errors for an off-axis static eye and for a rotating eye, and assigns certain weights to each of them.

\section{Disclosure}

The authors report no conflicts of interest.

\section{Acknowledgements}

This work was supported by grant FIS2016-75891-P awarded to Sergio Barbero and grant SFRH/BPD/116351/2016, funded by the Foundation for Science and Technology (FCT), awarded to Miguel Faria-Ribeiro

\section{References}


1. Holden BA, Fricke TR, Wilson DA, et al. Global prevalence of myopia and high myopia and temporal trends from 2000 through 2050. Ophthalmology 2016; 123: 10361042.

2. Flitcroft DI. The complex interactions of retinal, optical and environmental factors in myopia aetiology. Prog Retin Eye Res 2012; 31: 622-660.

3. Verkicharla PK, Ohno-Matsui K \& Saw SM. Current and predicted demographics of high myopia and an update of its associated pathological changes. Ophthalmic Physiol Opt 2015; 35: 465-475.

4. Wildsoet CF. Active emmetropization--evidence for its existence and ramifications for clinical practice. Ophthalmic Physiol Opt 1997; 17: 279-290.

5. Smith EL, Hung LF, Huang J, Blasdel TL, et al. Effects of optical defocus on refractive development in monkeys: evidence for local, regionally selective mechanisms. Invest Ophthalmol Vis Sci 2010; 51: 3864-3873.

6. Atchison DA, Li S-M, Li H, et al. Relative peripheral hyperopia does not predict development and progression of myopia in children. Invest Ophthalmol Vis Sci 2015; 56: 6162-6170.

7. Gonzalez-Meijome JM, Faria-Ribeiro MA, Lopes-Ferreira DP, et al. Changes in peripheral refractive profile after orthokeratology for different degrees of myopia. Current Eye Research 2016; 41: 199-207.

8. Faria-Ribeiro M, Navarro Belsue R, Lopez-Gil N \& Gonzalez-Meijome JM. Morphology, topography, and optics of the orthokeratology cornea. Journal of Biomedical Optics 2016; 21: 075011-075011.

9. Paune J, Queiros A, Lopes-Ferreira D, Faria-Ribeiro M, et al. Efficacy of a gas permeable contact lens to induce peripheral myopic defocus. Optom Vis Sci 2015; 92: 596-603.

10. Gonzalez-Meijome JM, Peixoto-de-Matos SC, Faria-Ribeiro M, et al. Strategies to regulate myopia progression with contact lenses: a review. Eye \& Contact Lens 2016; 42: 24-34.

11. Tabernero J, Vazquez D, Seidemann A, Uttenweiler D \& Schaeffel F. Effects of myopic spectacle correction and radial refractive gradient spectacles on peripheral refraction. Vision Res 2009; 49: 2176-2186.

12. Lin Z, Martinez A, Chen X, et al. Peripheral defocus with single-vision spectacle lenses in myopic children. Optom Vis Sci 2010; 87: 4-9.

13. Smith G, Atchison DA, Avudainayagam C \& Avudainayagam K. Designing lenses to correct peripheral refractive errors of the eye. J Opt Soc Am A-Opt Image Sci Vis 2002; 19: 10-18.

14. Smith EL, III, Greeman N, Greeman P, Ho A \& Holden BA. Methods and apparatuses for altering relative curvature of field and positions of peripheral, off-axis focal positions. 2006, U.S. Patent 7,025,460

15. Ho A, Smith EL, III, Padmaja S \& Holden BA. Method and apparatus for controlling peripheral image position for reducing progression of myopia. US Patent 2007/0159601. 2007.

16. Varnas SR. Ophthalmic lens element. US Patent: $2011 / 0037944$.

17. Varnas SR, Fisher SW \& Spratt RS. Ophthalmic lens element for myopia correction. US Patent: 2009/0257026: Carl Zeiss Vision Australia.

18. Atchison DA, Mathur A \& Varnas SR. Visual performance with lenses correcting peripheral refractive errors. Optom Vis Sci 2013; 90: 1304-1311.

19. Atchison DA. Third-Order theory of spectacle lenses applied to correction of peripheral refractive errors. Optom Vis Sci 2011; 88: 227-233.

20. Landgrave JEA \& MoyaCessa JR. Generalized Coddington equations in ophthalmic lens design. JOSA-A 1996; 13: 1637-1644.

21. Atchison DA. Spectacle lens design: Development and present state. Aust J Optom 1984; 67: 97-107. 
22. Rojo P, Royo S, Caum J, Ramírez J \& Madariaga I. Generalized ray tracing method for the calculation of the peripheral refraction induced by an ophthalmic lens. Opt Eng 2015; 54: 025106-025106.

23. Alonso J, GomezPedrero JA \& Bernabeu E. Local dioptric power matrix in a progressive addition lens. Ophthalmic Physl Opt 1997; 17: 522-529.

24. Osuagwu UL, Suheimat M, Wolffsohn JS \& Atchison DA. Peripheral refraction validity of the Shin-Nippon SRW5000 autorefractor. Optom Vis Sci 2016; 93: 12541261.

25. Faria-Ribeiro M, Queiros A, Lopes-Ferreira D, Jorge J \& Gonzalez-Meijome JM. Peripheral refraction and retinal contour in stable and progressive myopia. Optom and Vis Sci 2013; 90: 9-15.

26. Thibos LN, Wheeler W \& Horner D. Power vectors: an application of Fourier analysis to the description and statistical analysis of refractive error. Optom Vis Sci 1997; 74: 367-375.

27. Liu J, Miñano JC \& Benítez P. Double aspheric imaging design with unconstrained object to image mapping. Opt Express 2015; 23: 13370-13375.

28. Liu J, Benítez $P$ \& Miñano JC. Single freeform surface imaging design with unconstrained object to image mapping. Opt Express 2014; 22: 30538-30546.

29. Barbero S. Minimum tangential error ophthalmic lens design without multiparametric optimization. Opt Commun 2012; 285: 2769-2773.

30. Harris, W. F. Curvature of ellipsoids and other surfaces. Ophthalmic Physl Opt 2006; 26: 497-501.

31. Uemura $T$, Arai $Y$ \& Shimazaki $C$. Eye-Head coordination during lateral gaze in normal subjects. Acta Oto-Laryngol 1980; 90: 191-198.

32. Jalie M. The principles of ophthalmic lenses: London: Association of British Dispensing Opticians; 1984.

33. Vazquez D, Seidemann A, Altheimer H, Schaeffel F \& Uttenweiler D. Optical tracking of head movement patterns when wearing spectacle lenses with different radial power profiles. ARVO Annual Meeting; 2009:E-abstract 3981.

34. Han Y, Ciuffreda KJ, Selenow A, Bauer E, Ali SR \& Spencer W. Static aspects of eye and head movements during reading in a simulated computer-based environment with single-vision and progressive lenses. Invest Ophthalmol Vis Sci 2003; 44: 145153.

35. Curcio CA \& Allen KA. Topography of ganglion cells in human retina. J Comp Neurol 1990; 300: 5-25.

36. Wang YZ, Thibos LN \& Bradley A. Effects of refractive error on detection acuity and resolution acuity in peripheral vision. Invest Ophthalmol Vis Sci 1997; 38: 2134-2143.

37. Tabernero J, Ohlendorf A, Fischer MD, Bruckmann AR, Schiefer U \& Schaeffel F. Peripheral refraction profiles in subjects with low foveal refractive errors. Optom Vis Sci 2011; 88: 388-394

38. Atchison DA, Pritchard N \& Schmid KL. Peripheral refraction along the horizontal and vertical visual fields in myopia. Vision Res, 2006; 46: 1450-1458. 